\title{
Densidade, tamanho populacional e conservação de primatas em fragmento de Mata Atlântica no sul do Estado de Minas Gerais, Brasil
}

\author{
Maurício Djalles Costa ${ }^{1}$, Fernando Afonso Bonillo Fernandes ${ }^{1}$, Renato Richard Hilário², \\ Aline Vaz Gonçalves ${ }^{1}$ \& Janaína Maria de Souza ${ }^{1}$
}

1. Laboratório de Ecologia, Departamento de Biologia, Universidade do Vale do Sapucaí. Av. Prefeito Tuany Toledo, 470, Fátima, 37550-000 Pouso Alegre, MG. (mauriciodjalles@uai.com.br;
bonillofernandes@uol.com.br; aline_g16@yahoo.com.br; djananamaria@yahoo.com).
2. Centro de Ciências Exatas e da Natureza, Universidade Federal da Paraíba. Cidade Universitária, 58059-900 João Pessoa, PB. (renatohilario@gmail.com)

\begin{abstract}
Density, population size and primate conservation in Atlantic forest fragments in the south region of Minas Gerais, Brazil. The purpose of this work is to estimate the density and the population size of four primate species [Alouatta clamitans Cabrera, 1940; Callicebus nigrifrons (Spix, 1823); Callithrix aurita (É. Geoffroy, 1812); Cebus nigritus (Goldfuss, 1809)] which occur in a fragment of Atlantic forest of approximately 350 hectares located in Pouso Alegre, state of Minas Gerais, as well as to give subsidies for the conservation of those species in the area. The population surveying was carried out through the distance sampling method in linear transects (Distance Sampling). Data were collected between April and August 2008 from four transects deployed in the study area. The density and population size were calculated using the software Distance 5.0 and were estimated in $23,83 \pm 9,78$ ind. $/ \mathrm{km}^{2}$ for Callicebus nigrifrons, $14,76 \pm 5,92$ ind./ $/ \mathrm{km}^{2}$ for Callithrix aurita and 7,71 $\pm 2,13$ ind. $/ \mathrm{km}^{2}$ for Cebus nigritus. The population size was estimated in 83,0 $\pm 34,0$ individuals for $C$. nigrifrons, 52,0 $\pm 20,8$ individuals for Callithrix aurita and 27,0 7,4 individuals for Cebus nigritus. With regard to the howler monkey (A. clamitans), it was stated out that just a group with six individuals survive in the area. In conclusion, the chances for these isolated populations to survive are slim due to the risk of stochastic events. The creation of ecological corridors connecting the study area to the other fragments, besides the translocation of individuals from other areas of the Atlantic forest to this region, could provide alternatives to ensure the viability of these populations in a long-term. Therefore, it is necessary to consolidate public policies in Pouso Alegre that lead to the creation, enlargement and management of conservation units and incentives for the adoption of productive practices based on sustainability in these areas of ecological interest.
\end{abstract}

KEYWORDS. Alouatta clamitans, Callicebus nigrifrons, Callithrix aurita, Cebus nigritus, linear transects.

RESUMO. Este trabalho teve como objetivo estimar a densidade e o tamanho populacional de quatro espécies de primatas [Alouatta clamitans Cabrera, 1940; Callicebus nigrifrons (Spix, 1823); Callithrix aurita (É. Geoffroy, 1812); Cebus nigritus (Goldfuss, 1809)] que ocorrem em um fragmento de Mata Atlântica de aproximadamente 350 ha, localizado no município de Pouso Alegre, estado de Minas Gerais e reunir subsídios para a conservação dessas espécies na região. O levantamento populacional foi realizado através do método de amostragem de distâncias em transecções lineares (Distance Sampling). Os dados foram coletados entre os meses de abril e agosto de 2008 a partir de quatro transecções implantadas na área de estudo. Os cálculos de densidade e tamanho populacional foram realizados empregando-se o programa Distance 5.0. As densidades foram estimadas em 23,83 \pm 9,78 ind. $/ \mathrm{km}^{2}$ para Callicebus nigrifrons, $14,76 \pm 5,92 \mathrm{ind} . / \mathrm{km}^{2}$ para Callithrix aurita e 7,71 $\pm 2,13$ ind./ $/ \mathrm{km}^{2}$ para Cebus nigritus. O tamanho populacional foi estimado em 83,0 $\pm 34,0$ indivíduos para $C$. nigrifrons, 52,0 $\pm 20,8$ indivíduos para Callithrix aurita e 27,0 $\pm 7,4$ indivíduos para Cebus nigritus. Com relação ao bugio (A. guariba clamitans), constatou-se que apenas um grupo com seis indivíduos sobrevive na área. Concluise que, no caso de continuarem isoladas, essas populações têm poucas chances de sobrevivência no futuro frente aos riscos de eventos estocásticos. A criação de corredores ecológicos conectando a área de estudo aos outros fragmentos em seu entorno e a translocação de indivíduos de outras áreas da Mata Atlântica para esta região poderão constituir alternativas para garantir a viabilidade dessas populações em longo prazo. Para tanto, é necessário que se consolide uma política pública no município de Pouso Alegre voltada à criação, ampliação e gestão de Unidades de Conservação, e ao incentivo para a adoção de práticas produtivas sob critérios de sustentabilidade no entorno dessas áreas de interesse ecológico.

PALAVRAS-CHAVE. Alouatta clamitans, Callicebus nigrifrons, Callithrix aurita, Cebus nigritus, transecções lineares.

A Mata Atlântica é uma das regiões biologicamente mais ricas e mais ameaçadas do Planeta (MitTERmeier et al., 2005). Atualmente restam entre $11,4 \%$ e $16 \%$ de cobertura vegetal de sua extensão original. Este bioma apresenta diferentes fitofisionomias, das quais a Floresta Estacional Semidecidual é a segunda mais devastada, estando atualmente representada por fragmentos de pequeno tamanho e com alto grau de isolamento (RIBEIRo et al., 2009).

O município de Pouso Alegre, localizado no sul do Estado de Minas Gerais abriga, atualmente, somente $3 \%$ de remanescentes de Floresta Semidecidual em seu território. Originalmente as florestas se estendiam por todo o município numa área de aproximadamente 54.400 ha (SOS MatA AtLÂNTICA, 2009).

A diminuição e fragmentação de habitats florestais afetam várias espécies, em especial os primatas neotropicais que possuem hábitos essencialmente arborícolas. Das 24 espécies de primatas que ocorrem na Mata Atlântica, 15 encontram-se enquadradas em alguma categoria de ameaça segundo a lista oficial do Ministério do Meio Ambiente (MACHADO et al., 2005).

No município de Pouso Alegre ocorrem quatro espécies de primatas: Alouatta clamitans Cabrera, 1940; Callicebus nigrifrons (Spix, 1823); Callithrix aurita (É. Geoffroyi, 1812) e Cebus nigritus (Goldfuss, 1809) (Costa et al., no prelo). Destas, apenas o sagui-da-serraescuro ( $C$. aurita) é considerado ameaçado de extinção, muito embora as outras espécies sejam consideradas como "quase ameaçadas" (MACHADO et al., 2005). Diante disto, torna-se necessário o desenvolvimento de estudos que avaliem o estado de conservação e a viabilidade das populações desses primatas na região.

Neste contexto, este trabalho teve como objetivos estimar a densidade e o tamanho populacional de primatas em um fragmento de Mata Atlântica Semidecídua em Pouso Alegre, MG, e reunir subsídios para a conservação dessas espécies na região. 


\section{MATERIAIS E MÉTODOS}

Este estudo foi realizado em um fragmento de Mata Atlântica localizado em um complexo montanhoso denominado Serra de Santo Antônio, no município de Pouso Alegre, sul do Estado de Minas Gerais, Brasil. A região de estudo tem como referência as coordenadas geográficas $22^{\circ} 13^{\prime} \mathrm{S}$ e $45^{\circ} 58^{\prime} \mathrm{O}$ e é constituída por aproximadamente 350 ha de Floresta Estacional Semidecidual Montana. A área de estudo engloba o Parque Natural Municipal de Pouso Alegre (PNMPA) com cerca de 178 ha, a Reserva Particular do Patrimônio Natural Mata dos Sabiás e a Reserva Florestal do $14^{\circ}$ Grupo de Artilharia de Campanha do Exército Brasileiro (Fig. 1).

O clima na região é do tipo $\mathrm{Cwb}$, com verões chuvosos brandos e invernos secos conforme critérios definidos por KöPpen (1936). O índice pluviométrico anual situa-se entre 1.300 e $1.700 \mathrm{~mm}$ (COSTA et al., no prelo).

O levantamento populacional de primatas foi realizado através do método de amostragem de distâncias em transecções lineares (Distance Sampling) (Burnham et al., 1980; Buckland et al., 1993; Peres, 1999).

Para realizar as amostragens, foram implantadas quatro transecções com 1.014 m, 1.086 m, 1.170 m e 765 m de comprimento no interior do PNMPA. Tais transecções foram orientadas, paralelamente, no sentido norte/sul, sendo a distância entre elas de aproximadamente $300 \mathrm{~m}$ (Fig. 1).

As transecções foram percorridas duas vezes por semana das $09 \mathrm{~h} 00$ às $12 \mathrm{~h} 00$ e das $13 \mathrm{~h} 00$ às $16 \mathrm{~h} 00$, entre os meses de abril e agosto de 2008. Em cada dia de amostragem, as quatro transecções foram percorridas apenas uma vez a uma velocidade média de $1 \mathrm{~km} / \mathrm{h}$. Não houve coleta de dados em dias chuvosos.

Conforme os animais eram avistados, registravase o número de indivíduos do grupo, a distância entre o observador e o primeiro animal avistado, bem como o ângulo de avistamento. A distância do observador ao animal avistado foi medida com um telêmetro/ hipsômetro modelo 100LHA da Opti-logic (amplitude: 4-100 m; precisão: $0,5 \mathrm{~m}$ ) e um telêmetro modelo Yardage Pro 1000 da Bushnell (amplitude: 18-1.000 m; precisão: $0,9 \mathrm{~m})$. O ângulo de observação $\left(0-90^{\circ}\right)$ foi obtido com uma bússola magnética.

O programa Distance 5.0 (Тномаs et al., 2005) foi utilizado para realizar os cálculos de densidade e tamanho populacional. Simulações foram efetuadas com todos os modelos e ajustes disponíveis no programa com o objetivo de encontrar uma função de detecção que melhor se ajustasse aos dados. A escolha dos modelos foi conduzida levando em consideração os menores valores de AIC (Akaike Information Criterion) e de CV\% (Coeficientes de Variação).

\section{RESULTADOS}

Foram percorridos $164 \mathrm{~km}$ em 41 dias de amostragem e contabilizados 85 avistamentos, sendo quatro de Alouatta clamitans, 30 de Callicebus nigrifrons, 28 de Callithrix aurita e 23 de Cebus nigritus. Em virtude do baixo número de avistamentos de Alouatta clamitans, decidiu-se não realizar a estimativa

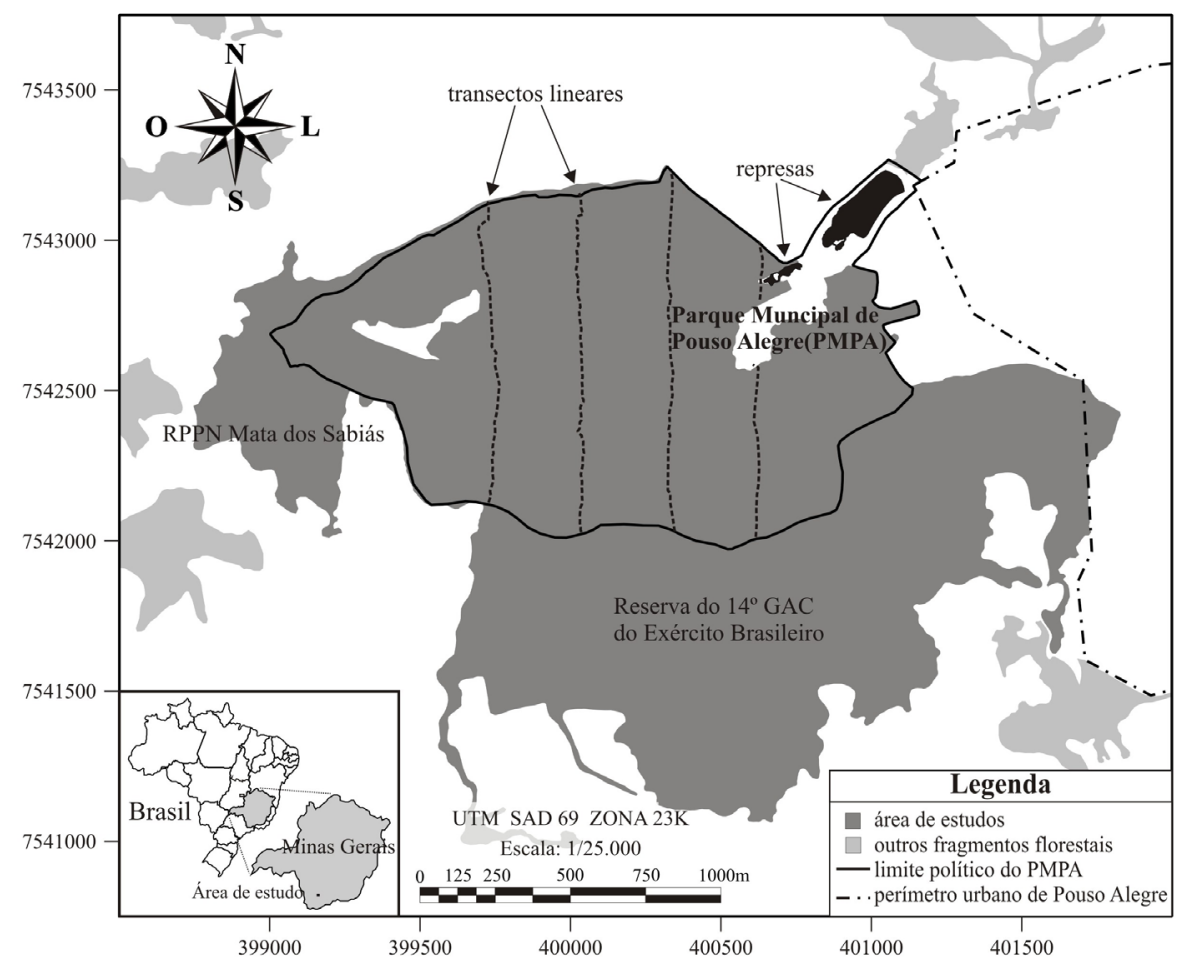

Fig. 1. Área de estudo com a definição dos transectos lineares em fragmento de Mata Atlântica, sul do estado de Minas Gerais, Brasil. 
de densidade e tamanho populacional desta espécie, tendo em vista que estimativas de densidade geradas a partir de avistamentos reduzidos fornecem resultados pouco confiáveis e com altos valores de coeficientes de variação.

O número de indivíduos por avistamento variou de 2 a $6(3,25 \pm 1,89)$ para $A$. clamitans, 1 a $5(2,3 \pm$ 1,02) para $C$. nigrifrons, 1 a $8(3,25 \pm 1,85)$ para $C$. aurita e 1 a $11(4,82 \pm 3,31)$ para $C$. nigritus. Os modelos que melhor se ajustaram aos dados foram o do Cosseno Exponencial Negativo (Negative Exponential Cosine) para C. nigrifrons e C. aurita, e o do Cosseno Uniforme (Uniform Cosine) para C. nigritus. Callicebus nigrifrons e C. nigritus foram as espécies que apresentaram a maior e a menor densidade e tamanho populacional, respectivamente (Tab. I). No entanto, infere-se que $A$. clamitans seja a espécie menos abundante na área de estudo, tendo em vista o pequeno número de encontros registrados.

\section{DISCUSSÃO}

Densidade Populacional. O sauá (C. nigrifrons) foi a espécie que apresentou a maior densidade populacional na área de estudo. Dentre os estudos consultados (Tab. II), esta é a maior densidade populacional encontrada para a espécie na Mata Atlântica $\left(23,83\right.$ ind. $\left./ \mathrm{km}^{2}\right)$.

O bugio-ruivo (A. clamitans) foi a espécie menos avistada durante o levantamento e, provavelmente, é a menos abundante na área de estudo. Devido à proximidade dos locais onde $A$. clamitans foi observada, o mais provável é que os quatro avistamentos foram de um mesmo grupo. Supondo a existência de apenas um grupo de bugios no Parque Natural Municipal de Pouso Alegre, com seis indivíduos (avistamento com maior número de indivíduos), a densidade populacional estimada é de 3,37 ind. $/ \mathrm{km}^{2}$. Esta é a segunda menor densidade encontrada na literatura até o momento (Tab. II), sendo superior apenas à densidade encontrada na Serra de Paranapiacaba $\left(0,79\right.$ ind. $\left./ \mathrm{km}^{2}\right)$ (GonZÁLEs-Solis et al., 2001).

A densidade populacional do macaco-prego-preto (C. nigritus) na área de estudo foi considerada baixa $\left(7,71\right.$ ind.$\left./ \mathrm{km}^{2}\right)$. Contudo, este valor encontra-se dentro das variações de densidade registradas em diferentes localidades da Mata Atlântica (entre 0,9 e 66,2 ind. $/ \mathrm{km}^{2}$ ) (Tab. II).

A densidade populacional do sagui-da-serraescuro (C. aurita) foi considerada alta $\left(14,76 \mathrm{ind} . / \mathrm{km}^{2}\right)$ se comparada às estimativas de densidade da espécie disponíveis na literatura (Cosenza \& Melo, 1998; São Bernardo \& Galetti, 2004) (Tab. II).

Vários fatores podem influenciar a densidade populacional de uma espécie, dentre eles, a disponibilidade de recursos (STEVENSON, 2001), a dieta e a flexibilidade ecológica da espécie (RoBInson \& Redford, 1986; Stevenson, 2001), a competição por recursos (TERBORgh, 1983), a pressão predatória (StANFord, 1995), a caça ilegal e a fragmentação de habitats (Peres, 1997). Tais fatores variam entre diferentes localidades e influenciam diferentemente cada uma das espécies. Dessa forma, ao menos que mais estudos sejam conduzidos, não é possível definir precisamente as causas para as baixas densidades de A. clamitans e $C$. nigritus e as altas densidades de $C$ nigrifrons e C. aurita para a área de estudo.

Tamanho populacional e estratégias de conservação. A área de estudo é um pequeno fragmento florestal de 350 ha e mantém populações de quatro espécies de primatas (estimativa de 83 indivíduos de sauás, 52 indivíduos de saguis, 27 indivíduos de macacos prego e avistamento de 6 indivíduos de bugio). Estes tamanhos populacionais podem ser considerados pequenos e, caso permaneçam isoladas, estas populações podem ter poucas chances de sobrevivência no futuro, tendo em vista a vulnerabilidade das mesmas a eventos estocásticos como flutuações demográficas aleatórias, períodos de seca, incêndios, epidemias, depressão endogâmica e deriva gênica (Franklin \& Frankham, 1998; Lynch \& LANDE, 1998).

A definição de um tamanho mínimo de uma população capaz de se garantir em longo prazo (população mínima viável-PMV) é algo complexo e tem proporcionado um intenso debate entre pesquisadores (Franklin \& Frankham, 1998). Normalmente, os valores para populações mínimas viáveis costumam ser definidos em 500 indivíduos ou mais (FrankLIN, 1980; Franklin \& Frankham, 1998; Lynch \& Lande, 1998, Reed \& Bryant, 2000, Reed et al., 2003). Porém, estudos de Análise de Viabilidade Populacional - AVP, com três espécies de primatas ameaçados de extinção da Mata Atlântica reportam populações com 90 indivíduos de Brachyteles hypoxanthus (Kuhl, 1820) (STRIER, 1993-1994) e 150-300 indivíduos de Leontopithecus chrysomelas (Kuhl, 1820) e Cebus xanthosternus Wied, 1820 (Paglia, 2003) como viáveis em 100 anos.

Infelizmente, os dados disponíveis para as espécies que ocorrem na área de estudo ainda são escassos para permitir uma AVP confiável destas. Portanto, se torna

Tab. I. Largura efetiva do transecto (ESW), densidade de grupos e indivíduos, e tamanho estimado da população para as três espécies das quais se obteve um número satisfatório de avistamentos, Serra de Santo Antonio, Pouso Alegre, MG.

\begin{tabular}{|c|c|c|c|c|c|}
\hline Espécie & $\mathrm{N}$ & $\operatorname{ESW}(\mathrm{m})$ & $\begin{array}{c}\text { Densidade } \\
\left(\text { grupos } / \mathrm{km}^{2}\right)\end{array}$ & $\begin{array}{c}\text { Densidade } \\
\text { (indivíduos } / \mathrm{km}^{2} \text { ) }\end{array}$ & $\begin{array}{c}\text { Tamanho estimado da } \\
\text { população (indiv.) }\end{array}$ \\
\hline Callicebus nigrifrons & 30 & $8,82 \pm 19,56$ & $10,36 \pm 4,16$ & $23,83 \pm 9,78$ & $83,0 \pm 34,0$ \\
\hline Callithrix aurita & 28 & $13,01 \pm 23,74$ & $4,86 \pm 1,88$ & $14,76 \pm 5,92$ & $52,0 \pm 20,8$ \\
\hline Cebus nigritus & 23 & $35,00 \pm 0,00$ & $1,60 \pm 0,37$ & $7,71 \pm 2,13$ & $27,0 \pm 7,4$ \\
\hline
\end{tabular}


Tab. II. Densidade para as espécies de primatas avaliadas neste estudo em localidades da Mata Atlântica.

\begin{tabular}{|c|c|c|c|c|}
\hline Espécie & Área de Estudo & $\begin{array}{l}\text { Tamanho do } \\
\text { fragmento } \\
\left(\mathrm{km}^{2}\right)\end{array}$ & $\begin{array}{l}\text { Densidade } \\
\text { (ind } / \mathrm{km}^{2} \text { ) }\end{array}$ & Referência \\
\hline \multirow[t]{9}{*}{ Alouatta clamitans } & Parque Estadual Serra do Brigadeiro, MG & 132,1 & 7,5 & Cosenza \& Melo, 1998 \\
\hline & Serra de Paranapiacaba, SP & 1000 & $0,79(0,39-1,19)$ & GonZÁLES-Solis et al., 2001 \\
\hline & Estação Biológica de Caratinga, MG & 9,57 & $50,2(38,1-67,2)$ & Almeida-Silva et al., 2005 \\
\hline & Floresta Água Sumida, Barreiro Rico, SP & 2,4 & $10,42(9,0-12,85)$ & MARTINS, 2005 \\
\hline & Floresta Monal, Barreiro Rico, SP & 3,74 & $8,32(7,12-10,2)$ & MARTINS, 2005 \\
\hline & Floresta Sarã, Barreiro Rico, SP & 5,01 & $34,61(29,75-42,6)$ & Martins, 2005 \\
\hline & Floresta Viraeiro/Tabatinguera, Barreiro Rico, SP & 14,5 & $27,13(23,35-3,42)$ & MARTINS, 2005 \\
\hline & Reserva Biológica Poço das Antas, RJ & 50,71 & 44,1 & ARAúJo et al., 2008 \\
\hline & Reserva Biológica União, RJ & 29,27 & 42,1 & ARAúJo et al., 2008 \\
\hline \multirow[t]{6}{*}{ Callicebus nigrifrons } & Parque Estadual Serra do Brigadeiro, MG & 132,1 & 10,3 & Cosenza \& Melo, 1998 \\
\hline & Universidade Federal de Viçosa, MG & 0,75 & 14,86 & OLIVEIRA et al., 2003 \\
\hline & Mata São José, Rio Claro, SP & 2,3 & $3,5(2,8-4,6)$ & SÃo BERnARdo \& GALETTI, 2004 \\
\hline & Parque Estadual da Cantareira, SP & 79,2 & $12,21(8,45-17,63)$ & TREVELIN et al., 2007 \\
\hline & Floresta Paraíso, Viçosa, MG & 3,84 & $7,35(4,97-9,73)$ & SANTANA et al., 2008 \\
\hline & Parque Natural Municipal de Pouso Alegre, MG & 3,6 & $23,83(14,05-3,61)$ & Este estudo \\
\hline \multirow[t]{3}{*}{ Callithrix aurita } & Parque Estadual Serra do Brigadeiro, MG & 132,1 & 2,8 & Cosenza \& Melo, 1998 \\
\hline & Mata São José, Rio Claro, SP & 2,3 & $3,5(2,8-4,6)$ & SÃo Bernardo \& Galetti, 2004 \\
\hline & Parque Natural Municipal de Pouso Alegre, MG & 3,6 & $14,76(8,84-20,68)$ & Este estudo \\
\hline \multirow[t]{14}{*}{ Cebus nigritus } & Parque Estadual Serra do Brigadeiro, MG & 132,1 & 0,9 & Cosenza \& Melo, 1998 \\
\hline & Reserva M7/317, ES & 2,6 & $21,36(18,78-4,29)$ & Chiarello, 2000 \\
\hline & Reserva Putiri, ES & 2,1 & $11,45(10,06-3,02)$ & Chiarello, 2000 \\
\hline & Serra de Paranapiacaba, SP & 1000 & $5,31(3,26-7,36)$ & GonZÁLEs-Solis et al., 2001 \\
\hline & Mata São José, Rio Claro, SP & 2,3 & $24,5(19,6-32,6)$ & SÃo Bernardo \& Galetti, 2004 \\
\hline & Estação Biológica de Caratinga, MG & 9,57 & $28,9(23,3-36,0)$ & Almeida-SiLva et al., 2005 \\
\hline & Ilha de Anchieta, SP & 8,28 & $4,2(3,43-5,26)$ & BOVENDORP \& GALETTI, 2006 \\
\hline & Floresta Água Sumida, Barreiro Rico, SP & 2,4 & $22(19,0-27,13)$ & MARTINS, 2005 \\
\hline & Floresta Monal, Barreiro Rico, SP & 3,74 & $43,98(37,68-3,85)$ & MARTINS, 2005 \\
\hline & Floresta Sarã, Barreiro Rico, SP & 5,01 & $15,34(13,23-8,94)$ & MARTINS, 2005 \\
\hline & Floresta Viraeiro/Tabatinguera, Barreiro Rico, SP & 14,5 & $47,92(41,22-8,99)$ & MARTINS, 2005 \\
\hline & Reserva Biológica Poço das Antas, RJ & 50,71 & 66,2 & ARAúJO et al., 2008 \\
\hline & Reserva Biológica União, RJ & 29,27 & 34 & ARAÚJO et al., 2008 \\
\hline & Parque Natural Municipal de Pouso Alegre, MG & 3,6 & $7,71(5,38-9,84)$ & Este estudo \\
\hline
\end{tabular}

mais interessante do ponto de vista conservacionista não esperar até que tais dados estejam disponíveis e assumir que tais populações não são viáveis em longo prazo, partindo para estratégias de conservação que envolvam os outros fragmentos florestais da região.

Neste contexto, observa-se que numa área de entorno equivalente a $96 \mathrm{~km}^{2}$, existem 95 fragmentos florestais que variam em tamanho de um a 1.000 ha. Este mosaico de fragmentos (incluindo a área de estudo) cobre uma área de aproximadamente 2.300 ha (Fig. 2). Contudo, somente quatro fragmentos possuem área superior a 50 ha $(88,95,98$, e 1.000 ha) além da área de estudo.

A matriz da paisagem é composta por áreas de pastagens de origem antrópica entremeadas por loteamentos habitacionais em zonas de expansão urbana do município de Pouso Alegre.
A distância entre os maiores fragmentos da região (> 50 ha) varia de $40-600 \mathrm{~m}$ nos pontos de maior proximidade entre os mesmos. É possível, que a matriz da paisagem seja permeável para os primatas, já havendo um fluxo de animais entre estes fragmentos. Entretanto, a implantação de corredores ecológicos conectando estes fragmentos, potencializaria o fluxo de indivíduos (genes) entre as populações e evitaria que os primatas corressem riscos ao atravessarem a matriz da paisagem.

O fluxo de indivíduos entre esses fragmentos, em tese, aumentaria as chances de sobrevivência das espécies de primatas em longo prazo na região. Uma vez conectados, os fragmentos com área superior a 50 ha (incluindo a área de estudo) passariam a constituir um mosaico florestal com aproximadamente 1.700 ha (considerando áreas de corredores ecológicos). 


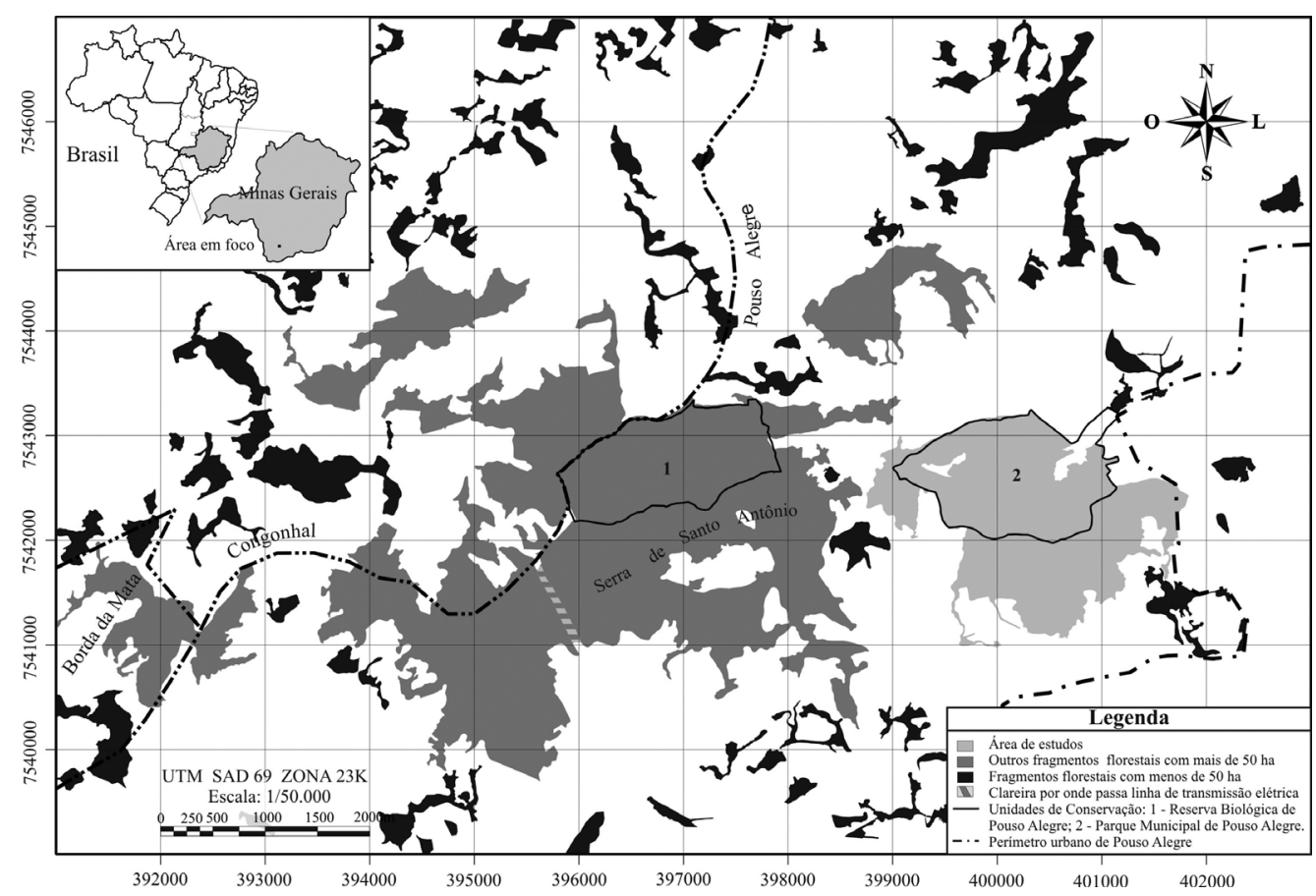

Fig. 2. Mapa com os fragmentos florestais no entorno da área de estudo, sul do estado de Minas Gerais, Brasil.

Levando em consideração as densidades populacionais reportadas neste estudo, este mosaico florestal poderia, teoricamente, abrigar populações, com $405 \pm 166$ indivíduos de C. nigrifrons, $250 \pm 100$ indivíduos de $C$. aurita e $131 \pm 36$ indivíduos de $C$. nigritus, o que aumentaria, consideravelmente, a chance dessas populações, principalmente de $C$. nigrifrons e $C$. aurita, persistirem ao longo de 100 anos.

Com relação aos bugios seria conveniente estimar a densidade destes animais nos remanescentes florestais no entorno da área de estudo, pelo menos no fragmento de 1.000 ha, pois caso também ocorra em baixas densidades, a estratégia mais plausível a fim de se evitar a extinção local da espécie num futuro próximo seria a translocação de indivíduos de outras áreas da Mata Atlântica para esta região.

Conclui-se que os primatas que ocorrem no Parque Natural Municipal de Pouso Alegre e entorno têm poucas chances de sobrevivência no futuro frente aos riscos de eventos estocásticos. A criação de corredores ecológicos conectando a área de estudo aos outros fragmentos do seu entorno e a translocação de indivíduos de outras áreas da Mata Atlântica para esta região poderão constituir alternativas para garantir a viabilidade dessas populações em longo prazo. Para que estas estratégias de conservação se concretizem, é necessário que se consolide uma política pública no município voltada à ampliação e criação de Unidades de Conservação, à preservação dos demais remanescentes de Mata Atlântica da região no contexto da ecologia da paisagem e ao incentivo de práticas produtivas sob critérios de sustentabilidade no entorno dessas áreas de interesse ecológico.
Agradecimentos. À Secretaria Municipal de Planejamento Urbano Sustentável, Meio Ambiente e Habitação de Pouso Alegre por ceder funcionários que ajudaram na abertura de trilhas no Parque Municipal de Pouso Alegre.

\section{REFERÊNCIAS BIBLIOGRÁFICAS}

Almeida-Silva, B.; Cunha, A. A.; Boubli, J. P.; Mendes, S. L. \& Strier, K. B. 2005. Population density and vertical stratification of four primate species at the Estação Biológica de Caratinga / RPPN-FMA, Minas Gerais, Brazil. Neotropical Primates 13 (suppl):25-29.

AraúJo, R. M.; Souza, M. B. \& Ruiz-Miranda, C. R. 2008. Densidade e tamanho populacional de mamíferos cinegéticos em duas Unidades de Conservação do Estado do Rio de Janeiro, Brasil. Iheringia, Série Zoologia 98(3):391-396.

Bovendorp, R. S. \& GaletTI, M. 2006. Density and population size of mammals introduced on a land-bridge island in southeastern Brazil. Biological Invasions 9(3):353-357.

Buckland, S. T.; Anderson, D. R.; Burnham, K. P. \& Laake, J. L. 1993. Distance sampling: estimating abundance of biological populations. London, Chapman and Hall. 432p.

Burnham, K. P.; Anderson, D. R. \& LaAke, J. L. 1980. Estimation of density from line transect sampling of biological populations. Wildlife Monographs 72:1-202.

Chiarello, A. G. 2000. Density and population size of mammals in remnants of Brazilian Atlantic Forest. Conservation Biology 14(6): 1649-1657.

Cosenza, B. A. P. \& Melo, F. R. 1998. Primates of the Serra do Brigadeiro State Park, Minas Gerais, Brazil. Neotropical Primates 6(1):18-20.

Costa, M. D.; Bonillo-Fernandes, F. A. \& Viana, D. H. S. (no prelo). Levantamento da mastofauna de médio e grande porte em fragmento de Mata Atlântica, Pouso Alegre, MG. Revista Brasileira de Zoociências.

Franklin, I. R. 1980. Evolutionary Change in Small Populations. In: Soulé, M. E. \& Wilcox, B. eds. Conservation Biology: evolutionary-ecological perspective. Sunderland, Sinaeur Associates. p.135-149

Franklin, I. R. \& Frankham, R. 1998. How large must population be to retain evolutionary potential? Animal Conservation 1:69-73.

GonzÁles-Solis, J.; Guix, J. C.; Mateos, E. \& Llorens, L. 2001. Population density of primates in a larger fragment of the Brazilian Atlantic Forest. Biodiversity and Conservation 10:1267-1282. 
KöPpen, W. 1936. Der Geographische System der Klima. In: KöpPeS, W. \& Geiger, R. eds. Handbuch der Klimatologie. Berlim, Borntrager. v. 1, part c.

Lynch, M. \& LANDE, R. 1998. The critical effective size for a genetically secure population. Animal Conservation 1:70-72.

Machado, A. B. M.; Martins, C. S. \& Drummond, G. M. 2005. Lista da Fauna Brasileira Ameaçada de Extinção: incluindo as listas das espécies Quase Ameaçadas e Deficientes em Dados. Belo Horizonte, Fundação Biodiversitas. 157p.

Martins, M. M. 2005. Density of primates in four semi-deciduous forest fragments of São Paulo, Brazil. Biodiversity and Conservation 14:2321-2329.

Mittermeier, R. A.; Valladares-Pádua, C.; Rylands, A. B.; Eudey, A. A.; Butynski, T. M.; Ganzhorn, J. U.; Kormos, R.; Aguiar, J. M. \& Walter, S. 2005. Primates in Peril: The World's 25 Most Endangered Primates 2004-2006. Washington DC, Report to IUCN/SSC Primate Specialist Group (PSG), International Primatological Society (IPS) and Conservation International (CI). $47 \mathrm{p}$.

Oliveira, R. C. R.; Coelho, A. S. \& Melo, F. R. 2003. Estimativa de densidade e tamanho populacional de sauá (Callicebus nigrifrons) em um fragmento de mata em regeneração em Viçosa, Minas Gerais, Brasil. Neotropical Primates 11(2):91-94.

Paglia, A. 2003. Análise de Viabilidade Populacional: Quantos indivíduos? Serão eles suficientes? Estudo de caso para espécies ameaçadas da Mata Atlântica do sul da Bahia. Corredor de Biodiversidade da Mata Atlântica do sul da Bahia. Belo Horizonte, Conservation International. (CD-room).

Peres, C. A. 1997. Effects of habitat quality and hunting pressure on arboreal folivore densities in Neotropical forest: a case study of howler monkeys (Alouatta spp.). Folia Primatologica 68:199-222.

.1999. General guidelines for standardizing line transect surveys of tropical forest primates. Neotropical Primates 7(1):11-16.

ReEd, D. H. \& Bryant, E. H. 2000. Experimental tests of minimum viable population size. Animal Conservation 3:7-14

Reed, D. H.; O'Grady, J. J.; Brook, B. W.; Ballou, J. D. \& Frankham, R. 2003. Estimates of minimum viable population sizes for vertebrates and factors influencing those estimates. Biological Conservation 113:23-34.
Ribeiro, M. C.; Metzger, J. P.; Martensen, A. M.; Ponzoni, F. J. \& Hirota, M. M. 2009. The Brazilian Atlantic Forest: How much is left, and how is the remaining forest distributed? Implications for conservation. Biological Conservation 142:1141-1153.

Robinson, J. G. \& RedFord, K. H. 1986. Body size, diet and population density of neotropical forest mammals. American Naturalist 128:665-680.

Santana, B. E. M. M.; Rocha, M. R.; Lessa, G.; Rocha, E. C. \& Melo, F. R. 2008. Densidade, tamanho populacional e abundância dos primatas em um fragmento de Floresta Atlântica em Minas Gerais, Brasil. Revista Árvore 32(6):1109-1117.

São Bernardo, C. S. \& Galetti, M. 2004. Densidade e tamanho populacional de primatas em um fragmento florestal no sudeste do Brasil. Revista Brasileira de Zoologia 21(4):827-832.

SOS Mata AtlÂNTICA. 2009. Atlas dos Remanescentes Florestais da Mata Atlântica. Disponível em: <http://mapas.sosma.org.br/>. Acesso em: 01.10.2009.

StANFORD, C. B. 1995. The influence of chimpanzee predation on group size and antipredator behaviour in red colobus monkeys. Animal Behaviour 49:577-587.

Stevenson, P. R. 2001. The relationship between fruit production and primate abundance in neotropical communities. Biological Journal of the Linnean Society 72:161-178.

StRIER, K. B. 1993-1994. Viability analyses of an isolated population of muriquis monkeys (Brachyteles arachnoides): implications for primate conservation and demography. Primate Conservation 14-15:43-52.

TERBORGH, J. 1983. Five new world primates: a study in comparative ecology. Princeton, Princeton University. 260p.

Thomas, L.; Laake, J. L.; Strindberg, S.; Marques, F. F. C.; Buckland, S. T.; Borchers, D. L.; Anderson, D. R.; Burnham, K. P.; Hedley, S. L.; Pollard, J. H.; Bishop, J. R. B. \& Marques, T. A. 2005. Distance 5.0. Release " $x$ "1. Research Unit for Wildlife Population Assessment, University of St. Andrews, UK. Disponível em: <http://www.ruwpa.st-and.ac.uk/distance/>. Acesso em: 05.04.2009.

Trevelin, L. C.; Port-Carvalho, M.; Silveira, M. \& Morell, E. 2007. Abundance, habitat use and diet of Callicebus nigrifrons Spix (Primates, Pitheciidae) in Cantereira State Park, São Paulo, Brazil. Revista Brasileira de Zoologia 24(4):1071-1077. 